\title{
Chromatic assimilation unaffected by perceived depth of inducing light
}

\author{
STEVEN K. SHEVELL AND DINGCAI CAO \\ Departments of Psychology and Ophthalmology and Visual Science, University of Chicago, Chicago
}

(Received September 7, 2003; ACCEPTED December 2, 2003)

\begin{abstract}
Chromatic assimilation is a shift toward the color of nearby light. Several studies conclude that a neural process contributes to assimilation but the neural locus remains in question. Some studies posit a peripheral process, such as retinal receptive-field organization, while others claim the neural mechanism follows depth perception,

figure/ground segregation, or perceptual grouping. The experiments here tested whether assimilation depends on a neural process that follows stereoscopic depth perception. By introducing binocular disparity, the test field judged in color was made to appear in a different depth plane than the light that induced assimilation. The chromaticity and spatial frequency of the inducing light, and the chromaticity of the test light, were varied. Chromatic assimilation was found with all inducing-light sizes and chromaticities, but the magnitude of assimilation did not depend on the perceived relative depth planes of the test and inducing fields. We found no evidence to support the view that chromatic assimilation depends on a neural process that follows binocular combination of the two eyes' signals.
\end{abstract}

Keywords: Chromatic induction, Chromatic assimilation, Stereo disparity, Figure/ground segregation

\section{Introduction}

The color appearance of a light depends not only on its spectral composition but also on other lights seen nearby. For example, a patch that appears yellow in the dark appears greenish against a uniform long-wavelength ("red") background (Shevell \& Wei, 1998). This is an instance of color contrast, which is a shift in appearance away from nearby light.

This paper focuses on a related but opposite phenomenon called chromatic assimilation, in which color perception shifts toward the appearance of nearby light. Whether nearby light causes contrast or assimilation depends on several factors, including its chromaticity, luminance, and spatial frequency (Helson, 1963; Fach \& Sharpe, 1986; Wyszecki, 1986).

Assimilation is described by von Bezold (1876) though relatively little research critically considers its physiological underpinnings. The eye's optics can cause assimilation with high spatial-frequency patterns. Spread light and chromatic aberration blur the retinal image (Marimont \& Wandell, 1994; Smith et al., 2001) and in consequence some light falls upon photoreceptors corresponding to a neighboring region of the scene. Most investigators, however, conclude that assimilation depends also on neural processes following transduction (Hurvich \& Jameson, 1974; DeValois \& DeValois, 1975; Fach \& Sharpe, 1986; de Weert \& van

Address correspondence and reprint requests to: Steven K. Shevell, Visual Science Laboratories, The University of Chicago, 940 East 57th Street, Chicago, IL 60637, USA. E-mail: shevell@uchicago.edu
Kruysbergen, 1987; King, 1988; Moulden et al., 1993; Monnier \& Shevell, 2003; Shevell \& Cao, 2003). These neural processes may be separated into peripheral (plausibly retinal) mechanisms, such as receptive-field organization (de Weert \& van Kruysbergen, 1987; Jameson \& Hurvich, 1989; de Weert, 1991), or more central (almost surely cortical) processes that mediate depth perception, figure/ground segregation, and perceptual grouping (Fuchs, 1923; Festinger et al., 1970; King, 1988; Taya et al., 1995; de Weert \& van Kruysbergen, 1997). Of course, optical, peripheral, and central processes all may contribute to assimilation.

Despite these studies, evidence for a central neural process of chromatic assimilation is not compelling. De Weert and van Kruysbergen (1997) provide a qualitative description but no data to support their claim that color appearance depends on the relative perceived depth of visual stimuli. Fuchs (1923), King (1988), and Taya et al. (1995) assume that assimilation occurs after perceptual grouping, an assumption not tested and at odds with the view that assimilation aids optimal perceptual segmentation and grouping by increasing the similarity of nearby objects (van Lier \& Wagemans, 1997).

The experiments here investigated whether assimilation involved a central neural process at or beyond the level of binocularly mediated depth perception. They determined how color appearance varied with the perceived relative depth of inducing and test lights. Initially, conditions for chromatic assimilation were established using lights perceived in a single depth plane. Then the relative perceived depth of the inducing light was changed by varying binocular disparity. The inducing light could appear either in front of or behind the test field judged in color. 
If assimilation is mediated by only optical factors and peripheral neural processes (before signals from the two eyes are combined), then the binocular disparity and thus relative depth of the test and inducing lights should not affect color perception. On the other hand, the figure/ground hypothesis of Festinger et al. (1970) and de Weert and van Kruysbergen (1997), which posits that assimilation is strongest in the perceived background, predicts the most assimilation when the inducing light is perceived nearer than the test field. Finally, the perceptual grouping (or "belonging") theory of assimilation (Fuchs, 1923; King, 1988) predicts assimilation is strongest when the test and inducing lights are coplanar, compared to when they are in different depth planes. Experiments here distinguish among these hypotheses.

\section{Materials and methods}

\section{Apparatus}

Stimuli were presented on a colorimetrically calibrated Sony 21inch video display, which was controlled by a Macintosh G4 computer with an auxiliary Radius video board (10 bits per gun). The resolution was $1360 \times 1024$ pixels and the refresh rate was $75 \mathrm{~Hz}$ noninterlaced. The stimuli were viewed through a haploscope (viewing distance $115 \mathrm{~cm}$ ) so that the left (right) half of the display was projected to only the left (right) eye. An adjustable chin rest was used to maintain a stable head position.

\section{Stimuli}

Separate test and comparison fields were viewed simultaneously. The test field judged in color and its inducing light, which caused assimilation, appeared above a uniform comparison field, which was adjusted by the observer to match the appearance of the test (Fig. 1a). The comparison field was a 2-deg-diameter circular disk. The test field was a circle of the same diameter but with inserted thin concentric rings composed of the inducing light. The inducing rings were characterized by their width and repetition rate. In this study, the rings were either $2 \mathrm{~min}$ of arc wide and repeated every $10 \mathrm{~min}$ of arc $(6$ cycles/deg) or $4 \mathrm{~min}$ of arc wide and repeated every $12 \mathrm{~min}$ of arc (5 cycles/deg). The outermost inducing ring had a diameter of $3 \mathrm{deg}$.

Experiments were conducted with test fields at $4 \mathrm{~cd} / \mathrm{m}^{2}$ and at two different chromaticities specified in MacLeod and Boynton (1979) $l, s$ units: $(0.665,0.99)$, which was nearly isomeric to equal energy white (EEW), and $(0.62,0.99)$, which appeared aqua. The unit of $s$ was arbitrary and scaled here to 1.0 for EEW. Inducing rings at $6 \mathrm{~cd} / \mathrm{m}^{2}$ were tested at two chromaticities: $(0.75,0.99)$, which appeared pinkish, and $(0.665,2.5)$, which appeared purplish.

The binocularly fused percept was the test with inserted inducing rings above the uniform comparison field. Binocular disparity was introduced by shifting the inducing rings relative to the test field judged in color (Fig. 1b). With 6 min of arc crossed disparity (both sets of inducing rings shifted toward the center of the scene), the inducing rings appeared in front of the test field; with 6 min of arc uncrossed disparity (rings shifted away from the center of the scene), the rings appeared behind the test. The perceived separation in depth between the rings and the test was conspicuous. With no disparity, the test and inducing rings were perceived in the same depth plane. The 3-deg diameter of the outermost inducing ring ensured that the 2-deg-diameter test and its nearby dark area were fully covered by inducing rings after the lateral shifts required for binocular disparity.

\section{Procedure}

The appearance of the test field with inserted inducing rings was measured by asymmetric matching. Following 3 min of dark adaptation, the observer adjusted the hue, saturation, and luminance of the comparison field to match the color appearance of the test. Adjustments were made via a multibutton game pad sensed by the computer. At the beginning of each trial, the comparison field was assigned a random starting chromaticity and luminance. Within
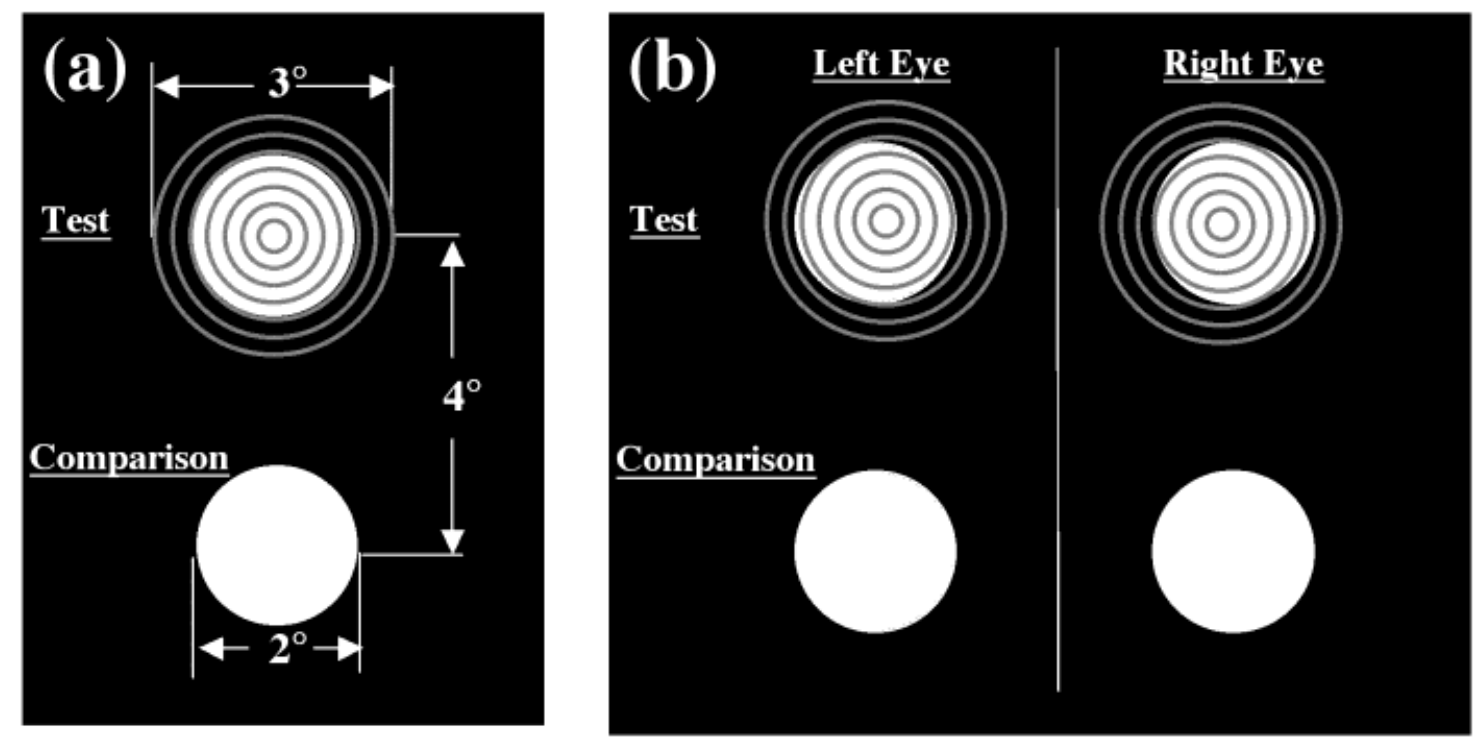

Fig. 1. (a) Schematic of the fused percept from haploscopically presented test and comparison fields. The inducing rings were varied in binocular disparity so they appeared nearer, farther away, or in the same depth plane as the test area. (b) Haploscopic stimuli presented to the left and right eyes. Crossed disparity is shown, which caused the inducing rings to appear nearer than the test field. 
a session, the size of inducing rings and the chromaticity of the test were fixed while the chromaticity of the inducing rings was varied. Binocular disparity was varied between sessions. Each condition was repeated four times within a session, in random order.

Each condition was repeated in four separate sessions on different days. The order of sessions was randomized. Standard errors were calculated using the mean value for each condition from each of the four sessions.

\section{Observers}

Three observers participated in the study. Two were naïve regarding the design and purpose of the experiments, and one was author D.C. All observers had normal color vision as determined by Rayleigh matching. They signed an informed consent form before participating in the experiments, which were approved by an Institutional Review Board at the University of Chicago.

\section{Results}

Matches to the color appearance of the test-with-inducing-rings showed clear chromatic assimilation. Measurements are shown in Fig. 2 for the pinkish inducing chromaticity $(l, s 0.75,0.99)$, which differed from EEW along only the $L /(L+M)$ dimension. Each panel shows results for a single observer for all combinations of test chromaticity (EEW or aqua) and inducing-ring size (width 2 or $4 \mathrm{~min}$ of arc). In these conditions, the test and inducing chromaticities were identical along the $S /(L+M)$ dimension $(s=0.99)$ but differed substantially in $L /(L+M)$. It was not surprising, therefore, that the assimilation caused by the inducing light was essentially along only the $L /(L+M)$ dimension. Measurements are shown, therefore, for only the $L /(L+M)$ value (vertical axis, Fig. 2).

Each open bar shows the match with no inducing light, for one of the combinations of test chromaticity and inducing-ring size (horizontal axis). Chromatic assimilation with inducing rings at chromaticity $(0.75,0.99)$ is indicated by a higher setting of $L /(L+M)$ than the value shown by the open bar. When the test and inducing lights were perceived in the same depth plane (no binocular disparity), the measurements showed significant chromatic assimilation for every observer in each condition (compare adjacent open and solid bars in Fig. 2; $P<0.001$ by sign test).

Crossed (uncrossed) disparity caused the inducing rings to appear nearer (farther away) than the test light but did not affect chromatic assimilation (stippled bars in Fig. 2). Measurements were very similar to those with no disparity. This too held for every observer in each condition. A separate analysis of variance for each observer (3-way crossed design, TEST CHROMATICITY $\times$ INDUCING-RING SIZE $\times$ DISPARITY) assessed whether the test chromaticity, inducing-ring size, and disparity affected the color matches. For every observer, the test chromaticity and inducing-ring size significantly changed the appearance of the test $(P \leq 0.04$ for each factor for every observer) but disparity or any interaction with disparity never approached statistical significance ( $P \geq 0.42$ for each of the four hypothesis tests that included disparity as a factor, for every observer).

Measurements with the other inducing-ring chromaticity $(0.665$, 2.5) are shown in Fig. 3. This inducing chromaticity differed from EEW along only the $s$ dimension, and match settings are shown only for $s$. With the EEW test field, which was identical to the inducing chromaticity in $l$ (0.665), the inducing light caused virtually no shift in $l$; with the aqua test $(0.62,0.99)$, assimilation
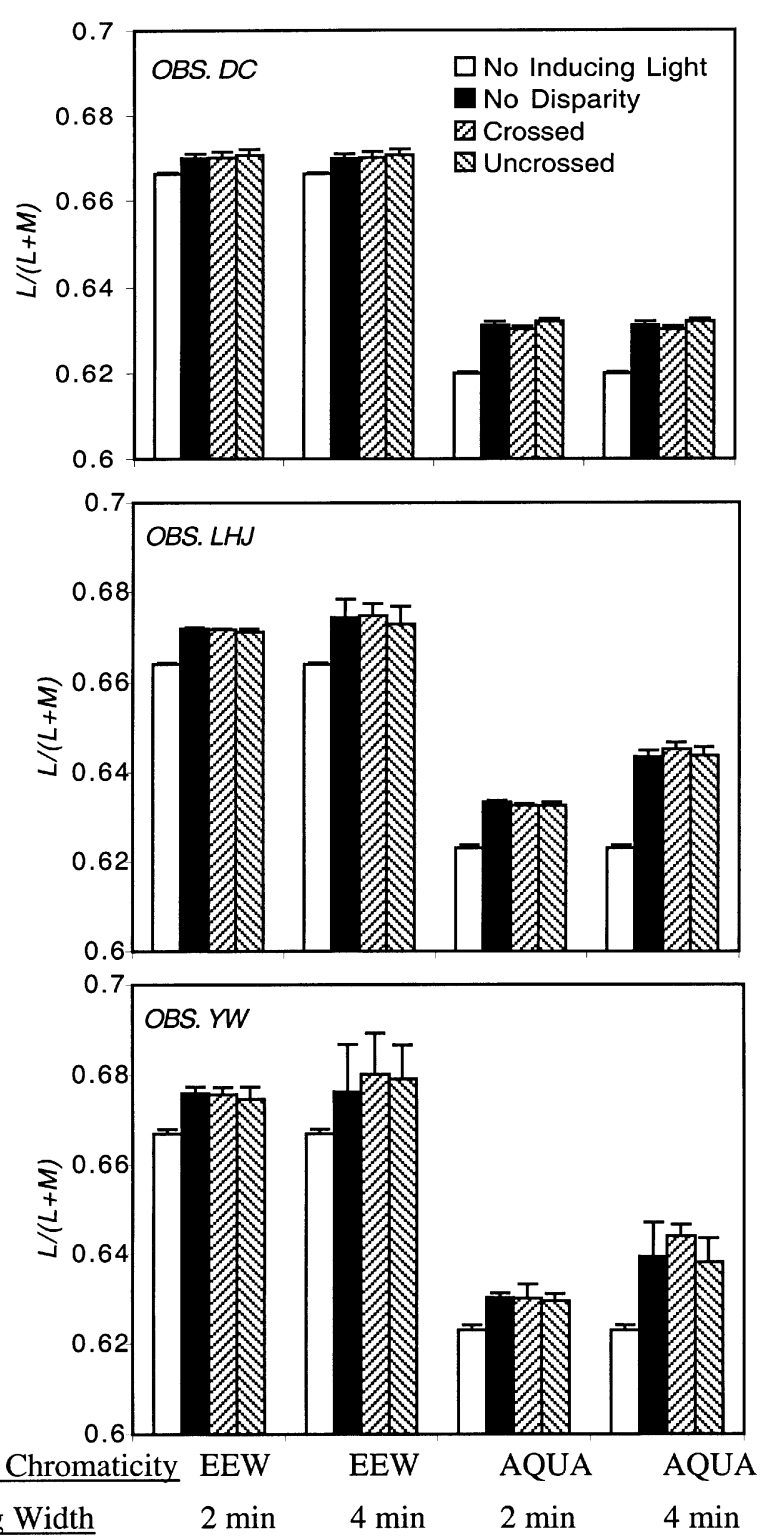

Fig. 2. Asymmetric matching measurements along the $L /(L+M)$ dimension to the color appearance of the test, presented with no inducing rings (open bar), inducing rings perceived in the same depth plane as the test (solid bar), or inducing rings perceived in a different depth plane than the test (stippled bars). The test chromaticity was either EEW or aqua ( $l, s$ $0.62,0.99)$; the inducing chromaticity was pinkish $(l, s 0.75,0.99)$. Inducingring width was either 2 min of arc or 4 min of arc. Each panel shows results for a different observer. Error bars indicate the standard error of the mean.

was observed in both the $s$ and $l$ directions with $l$ shifts (not shown) following the same pattern as the other results (Figs. $2 \& 3$ ). With no disparity, the inducing rings caused assimilation for each observer in every condition (compare each adjacent pair of open and solid bars in Fig. 3; $P<0.001$ by sign test). Introducing binocular disparity did not significantly change the color appearance of the test. Analysis of variance again showed for each observer a reliable effect of test chromaticity and inducing-ring size $(P \leq 0.03$ for each factor for every observer) but no significant effect of disparity or any interaction with disparity $(P \geq 0.14$ for all hypothesis tests that included disparity as a factor). 

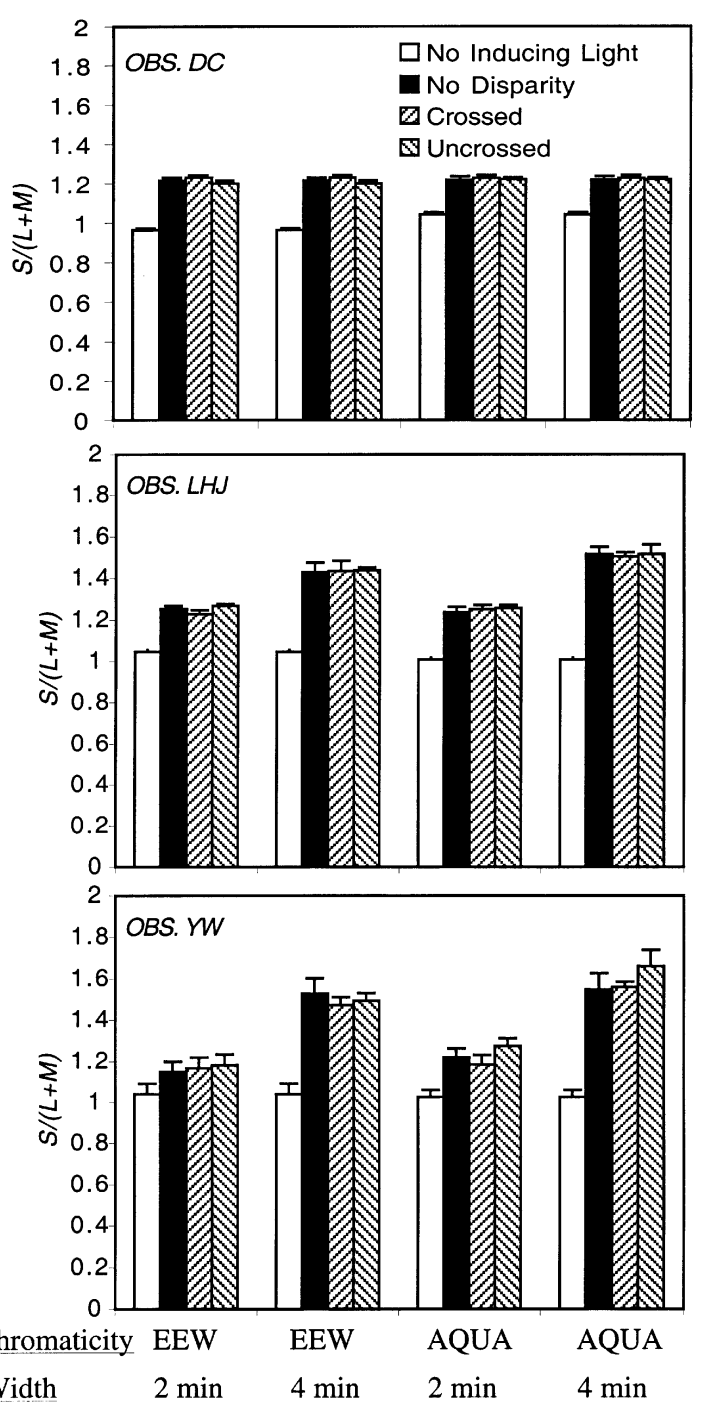

Fig. 3. Asymmetric matching measurements along the $S /(L+M)$ dimension to the color appearance of the test, presented with no inducing rings (open bar), inducing rings perceived in the same depth plane as the test (solid bar), or inducing rings perceived in a different depth plane than the test (stippled bars). The test chromaticity was either EEW or aqua $(l, s$ $0.62,0.99)$; the inducing chromaticity was purplish $(l, s 0.665,2.5)$. Inducingring width was either $2 \mathrm{~min}$ of arc or $4 \mathrm{~min}$ of arc. Each panel shows results for a different observer. Error bars indicate the standard error of the mean.

\section{Discussion}

Chromatic assimilation was observed with every inducing chromaticity, every inducing width ( 2 or 4 min of arc), every test chromaticity, and all stereo disparities (crossed, uncrossed, or none). The strength of assimilation depended on the chromaticities and on the width of the inducing light but not on the disparity. Further, no interaction between disparity and either test chromaticity or inducing-ring width was found, in any condition for any observer. While being mindful not to accept a null hypothesis, we found no significant change in chromatic assimilation due to the perceived relative depth of inducing and test lights.

Assimilation does depend on a neural process (Shevell \& Cao, 2003). Perceptual grouping, figure/ground segregation, and depth perception may influence assimilation in some situations, as claimed in previous studies (and see also Xian \& Shevell, 2004). Here, however, no empirical support was found for a central mechanism that follows binocular combination of neural signals from the two eyes.

\section{Acknowledgments}

This research was supported by PHS grant EY-04802. Publication was supported in part by an unrestricted grant to the Department of Ophthalmology and Visual Science from Research to Prevent Blindness.

\section{References}

DeValois, R.L. \& DeValois, K.K. (1975). Neural coding of color. In Handbook of Perception, Vol. 5, ed. Carterette, E.C. \& Friedman, M.P., pp. 117-166. New York: Academic Press.

DE WeERT C.M.M. (1991). Assimilation versus contrast. In From Pigments to Perception, ed. Valberg, A. \& Lee, B.B., pp. 305-311. New York: Plenum Press.

de Weert C.M.M. \& van Kruysbergen M.A.W.H. (1987). Subjective contour strength and perceptual superimposition: Transparency as a special case. In The Perception of Illusory Contours, eds S. Petry \& G. MeYer, pp. 165-170. New York: Springer,

De Weert, C.M.M. \& van Kruysbergen, M.A.W.H. (1997). Assimilation: Central and peripheral effects. Perception 26, 1217-1224.

FACH, C. \& Sharpe, L.T. (1986). Assimilative hue shifts in color gratings depend on bar width. Perception and Psychophysics 40, 412-418.

Festinger, L., Coren, S. \& Rivers, G. (1970). The effect of attention on brightness contrast and assimilation. American Journal of Psychology 83, 189-207.

FucHs, W. (1923). Experimentelle Untersuchungen über die Änderung von Farben unter dem Einfluss von Gestalten (Angleichungsercsheinungen) (Experimental investigations on the alteration of color under the influence of Gestalten). Zeitschrift für Psychologie 92, 249-325.

Helson, H. (1963). Studies of anomalous contrast and assimilation. Journal of the Optical Society of America 53, 179-184.

HuRvich, L.M. \& JAMESON, D. (1974). Opponent processes as a model of neural organization. American Psychologist 29, 88-102.

JAMESON, D. \& HURVICH, L.M. (1989). Essay concerning color constancy. Annual Review of Psychology 40, 1-22.

KING, D.L. (1988). Assimilation is due to one perceived whole and contrast is due to two perceived wholes. New Ideas in Psychology 6, 277-288.

MacLeod, D.I.A. \& Boynton, R.M. (1979). Chromaticity diagram showing cone excitation by stimuli of equal luminance. Journal of the Optical Society of America 69, 1183-1185.

Marimont, D.H. \& Wandell, B.A. (1994). Matching color images: The effects of axial chromatic aberration. Journal of the Optical Society of America A 12, 3113-3122.

Monnier, P. \& Shevell, S.K. (2003). Large shifts in color appearance from patterned chromatic backgrounds. Nature Neuroscience 6, 801-802.

Moulden, B., Kingdom, F. \& Wink, B. (1993). Colour pools, brightness pools, assimilation and the spatial resolving power of the human colour-vision system. Perception 22, 343-351.

Shevell, S.K. \& CAO, D. (2003). Chromatic assimilation: Evidence for a neural mechanism. In Normal and Defective Colour Vision, ed. MoLLON, J.D., Pokorny, J. \& Knoblauch, K., pp. 114-121. Oxford: Oxford University Press.

Shevell, S.K. \& WeI, J. (1998). Chromatic induction: Border contrast or adaptation to surrounding light? Vision Research 38, 1561-1566.

Smith, V.C., Jin, P. \& Pokorny, J. (2001). The role of spatial frequency in color induction. Vision Research 41, 1007-1021.

Taya, R., Ehrenstein, C. \& Cavonius, R. (1995). Varying the strength of the Munker-White effect by stereoscopic viewing. Perception 24, 685-693.

Van Lier, R. \& Wagemans, J. (1997). Perceptual grouping measured by color assimilation: Regularity versus proximity. Acta Psychologica 97, 37-70.

von Bezold, W. (1876). The Theory of Color and its Relation to Art and Art-Industry. Boston, Massachusetts: Prang.

Wyszecki, G. (1986). Color Appearance. In Handbook of Perception and Human Performance, Vol I: Sensory Processes and Perception, ed. Boff, K.R., Kaufman, L. \& Thomas, J.P., pp. 9-1-9-57. New York: Wiley.

Xian, S. \& Shevell, S.K. (2004). Changes in color appearance caused by perceptual grouping. Visual Neuroscience 21, 383-388. 